\title{
Effects of Endotoxin and Catecholamines on Hepatic Mitochondrial Respiration
}

\author{
Francesca Porta, ${ }^{1}$ Hendrik Bracht, ${ }^{1}$ Christian Weikert, ${ }^{1}$ Mario Beck, ${ }^{1}$ Jukka Takala, \\ Sebastian Brandt, ${ }^{2}$ Luzius B. Hiltebrand, ${ }^{2}$ and Stephan M. Jakob ${ }^{1,3}$
}

Abstract - Catecholamines are frequently used in sepsis, but their interaction with mitochondrial function is controversial. We incubated isolated native and endotoxin-exposed swine liver mitochondria with either dopamine, dobutamine, noradrenaline or placebo for $1 \mathrm{~h}$. Mitochondrial State 3 and 4 respiration and their ratio (RCR) were determined for respiratory chain complexes I, II and IV. All catecholamines impaired glutamate-dependent RCR $(p=0.046)$, predominantly in native mitochondria. Endotoxin incubation alone induced a decrease in glutamate-dependent RCR compared to control samples $(p=0.002)$. We conclude that catecholamines and endotoxin impair the efficiency of mitochondrial complex I respiration in vitro.

KEY WORDS: endotoxin; liver; mitochondria; catecholamines.

\section{INTRODUCTION}

Catecholamines are routinely used to support hemodynamics in patients with shock. In native hepatic mitochondria exposed to dopamine, increased oxygen loss as a consequence of membrane swelling has been demonstrated [4]. Furthermore, dopamine may induce ATP and glutathione depletion due to quinine formation and production of reactive oxygen species in hepatocytes [7]. On the other hand, noradrenaline increases the activity of hepatic succinate dehydrogenase [24], and adrenaline enhances the activity of mitochondrial phosphorylation and ATP production, resulting in increased

This work should be attributed to the Bern University Hospital and University of Bern, CH-3010 Bern, Switzerland

\footnotetext{
${ }^{1}$ Department of Intensive Care Medicine, Inselspital, Bern University Hospital and University of Bern, Freiburgstrasse, 3010, Bern, Switzerland

${ }^{2}$ Department of Anesthesiology and Pain Therapy, Inselspital, Bern University Hospital and University of Bern, Freiburgstrasse, 3010, Bern, Switzerland

${ }^{3}$ To whom correspondence should be addressed at Department of Intensive Care Medicine, Inselspital, Bern University Hospital and University of Bern, Freiburgstrasse, 3010, Bern, Switzerland. E-mail: stephan.jakob@insel.ch
}

oxygen consumption [1]. Reductions in mitochondrial enzyme function have been described as a consequence of selective beta adrenergic blockade [20] and after reserpin-induced depletion of catecholamines [23].

In a recent in vitro study, dopamine and dobutamine did not alter native muscle mitochondrial respiration but restored partially LPS-induced muscle mitochondrial uncoupling [21]. On the other hand, the combination of increased hepatosplanchnic oxygen delivery and decreased oxygen consumption in septic patients [12] raises the question of whether these drugs might worsen cellular homeostasis in the liver.

The aim of this study was to evaluate the effects of different catecholamines on respiration of isolated native and endotoxin-exposed liver mitochondria.

\section{MATERIALS AND METHODS}

\section{Anesthesia and Monitoring}

The study was performed in accordance with the National Institutes of Health guidelines for the care and use of experimental animals and with the approval of the 
Animal Care Committee of the County of Berne, Switzerland.

The animals used in this study served as controls for other studies published in abstract form and focusing on peritoneal membrane microdialysis [15] and splanchnic vascular reactivity in sepsis $[11,25]$.

Six pigs $(37-42 \mathrm{~kg})$ were deprived of food but not water for $24 \mathrm{~h}$ before the experiments. They were premedicated intramuscularly with atropine $0.05 \mathrm{mg} / \mathrm{kg}$ body weight, xylazine $2 \mathrm{mg} / \mathrm{kg}$ and ketamine $20 \mathrm{mg} / \mathrm{kg}$, followed 10 min later by cannulation of an ear vein and intravenous administration of $5-15 \mathrm{mg} / \mathrm{kg}$ thiopentone for endotracheal intubation. Anesthesia was maintained with thiopental $(5 \mathrm{mg} / \mathrm{kg} / \mathrm{h})$ and fentanyl $(5 \mu \mathrm{g} / \mathrm{kg} / \mathrm{h})$. Twelve hours later liver biopsies were excised and immediately transferred to $100 \mathrm{ml}$ ice-cold buffer solution for transport to the analytical laboratory. At the end of the experiment, the animals were sacrificed with an overdose of intravenous potassium chloride.

\section{Liver Mitochondrial Isolation}

Isolation of liver mitochondria was performed at $4^{\circ} \mathrm{C}$ using a standard procedure based on differential centrifugation [13]. The liver samples (6-8 g) were rapidly immersed in ice-cold isolation buffer (mannitol $220 \mathrm{mmol} / \mathrm{l}$, sucrose $70 \mathrm{mmol} / \mathrm{l}$, morpholinopropane sulfonic acid $5 \mathrm{mmol} / \mathrm{l}, \mathrm{pH}$ 7.4), transported to the laboratory, and weighed. Tissue was minced with scissors and homogenized with additional homogenization media (isolation buffer plus ethylene glycol-tetra-acetate $2 \mathrm{mmol} / \mathrm{l}$ ) 10 volumes (wt/vol) in a Potter Elvehjem homogenizer with a loose-fitting Teflon pestle (four strokes). The homogenate was then centrifuged for $10 \mathrm{~min}$ at $700 \times g$. The supernatant was collected and centrifuged again for $10 \mathrm{~min}$ at $7,000 \times \mathrm{g}$. At this time, the supernatant was discarded; the pellet was resuspended in isolation buffer and centrifuged twice for $10 \mathrm{~min}$ at $7,000 \times g$, for further purification of the mitochondria. The pellets were then suspended in buffer at a final concentration of $50-100 \mathrm{mg}$ of mitochondrial protein per $\mathrm{ml}$.

\section{Determination of Mitochondrial Respiration}

To determine the respiratory function, mitochondria were incubated in a $3 \mathrm{ml}$ incubation chamber (Yellow Springs Instruments, Yellow Springs, OH, USA) at $37^{\circ} \mathrm{C}$, in a medium consisting of $\mathrm{KCL} 25 \mathrm{mmol} / \mathrm{l}$, morpholinopropane sulfonic acid $12.5 \mathrm{mmol} / \mathrm{l}$, ethylene glycol-bis $\mathrm{N}, \mathrm{N}, \mathrm{N}^{\prime}, \mathrm{N}^{\prime}$-tetraacetic acid $1 \mathrm{mmol} / \mathrm{l}$, and phosphate buffer $5 \mathrm{mmol} / \mathrm{l}, \mathrm{pH}$ 7.4. Oxygen consumption was determined using a Clark type electrode
(Yellow Springs Instruments, Yellow Springs, OH, USA) in the presence of glutamate $20 \mathrm{mmol} / 1$ to examine complex I-dependent respiration, succinate $20 \mathrm{mmol} / \mathrm{l}$ for complex II-dependent respiration and ascorbate $0.12 \mathrm{mmol} / \mathrm{L} / \mathrm{N}, \mathrm{N}, \mathrm{N}^{\prime}, \mathrm{N}^{\prime}$-tertamethyl-p-phenyldiamine (TMPD) $0.24 \mathrm{mmol} / \mathrm{l}$ for complex IV-dependent respiration.

As shown in Fig. 1, mitochondrial respiratory function is conventionally separated into different states [8]. State 3 is defined as ADP-dependent oxygen consumption, and reflects the mitochondrial respiration coupled with ATP production. State 4, the resting respiration, is a measure of the oxygen consumed uncoupled from ATP synthesis, but required to maintain the integrity of the membrane potential.

We determined state 3 respiration rates in the presence of adenosine diphosphate (ADP) $200 \mu \mathrm{mol} / \mathrm{l}$. The rates measured after the consumption of ADP were taken as the state 4 respiration rates. Oxygen consumption rates are expressed as nanoatom $\mathrm{O}_{2} / \mathrm{min} / \mathrm{mg}$ protein.

Respiratory control ratios (RCR: State 3/State 4) for all complexes and ADP:O ratios (ADP added/oxygen consumed, nanomol/nanoatom) for complexes I to II were calculated according to Estabrook [10]. Finally, the maximal ATP production (ADP:O ratio $\times$ State 3 respiration, nanomol $\times$ nanoatom $/ \mathrm{min} / \mathrm{mg}$ protein) for complexes I and II was derived [17].

As shown in Fig. 1, the oxygen concentration decreases during measurement of complex respiration. Since the oxygen concentration in the oxygen buffer is $159 \mathrm{mmHg}$ at $100 \%$, it is unlikely that oxygen

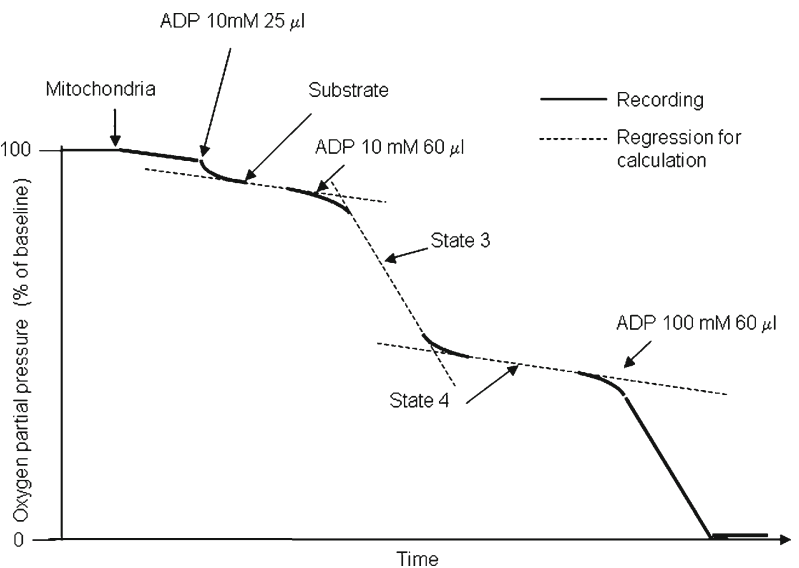

Fig. 1. Scheme of mitochondrial respiration analysis. State 3 represents oxygen use in the presence of ADP. State 4 represents oxygen use after ADP is completely transformed to ATP. At $37^{\circ} \mathrm{C} 100 \%$ oxygen saturation corresponds to a $\mathrm{PaO}_{2}$ of $159 \mathrm{mmHg}$. 
availability influenced the results. However, in order to avoid an influence, for the statistical analysis we always included the first curve we obtained after addition of substrates and ADP; in particular during glutamatedependent measurements, state 3 and 4 rates were included in the first $30 \%$ of oxygen decrease, approaching a $\mathrm{PaO}_{2}$ of $100 \mathrm{mmHg}$.

\section{Experimental Protocol}

Mitochondria from each biopsy sample were divided into eight groups and incubated on ice. Four groups of mitochondria were supplemented with endotoxin (LPS, Escherichia coli lipopolysaccharide B0111: B4, Difco Laboratories, Detroit, MI, USA) at a concen- tration of $100 \mu \mathrm{g} / \mathrm{mg}$ mitochondrial protein, and four groups were supplemented with placebo (isolation buffer) at the same concentration. After $1 \mathrm{~h}$ of incubation with endotoxin or placebo on ice, either dopamine, dobutamine (both at final concentrations of $100 \mu \mathrm{M}$ ), noradrenaline (at a final concentration of $60 \mu \mathrm{M}$ ) or placebo was added for one additional hour to both the endotoxin and placebo groups. Tyrosinase was added at a final concentration of $100 \mathrm{U} / \mathrm{ml}$. Stocks of LPS, dopamine, dobutamine and noradrenaline were diluted in isolation buffer before being added to the mitochondria. The effects of LPS and placebo alone were tested after $2 \mathrm{~h}$ of incubation.

The protocol was started immediately after isolation of the mitochondria, and the determination of the
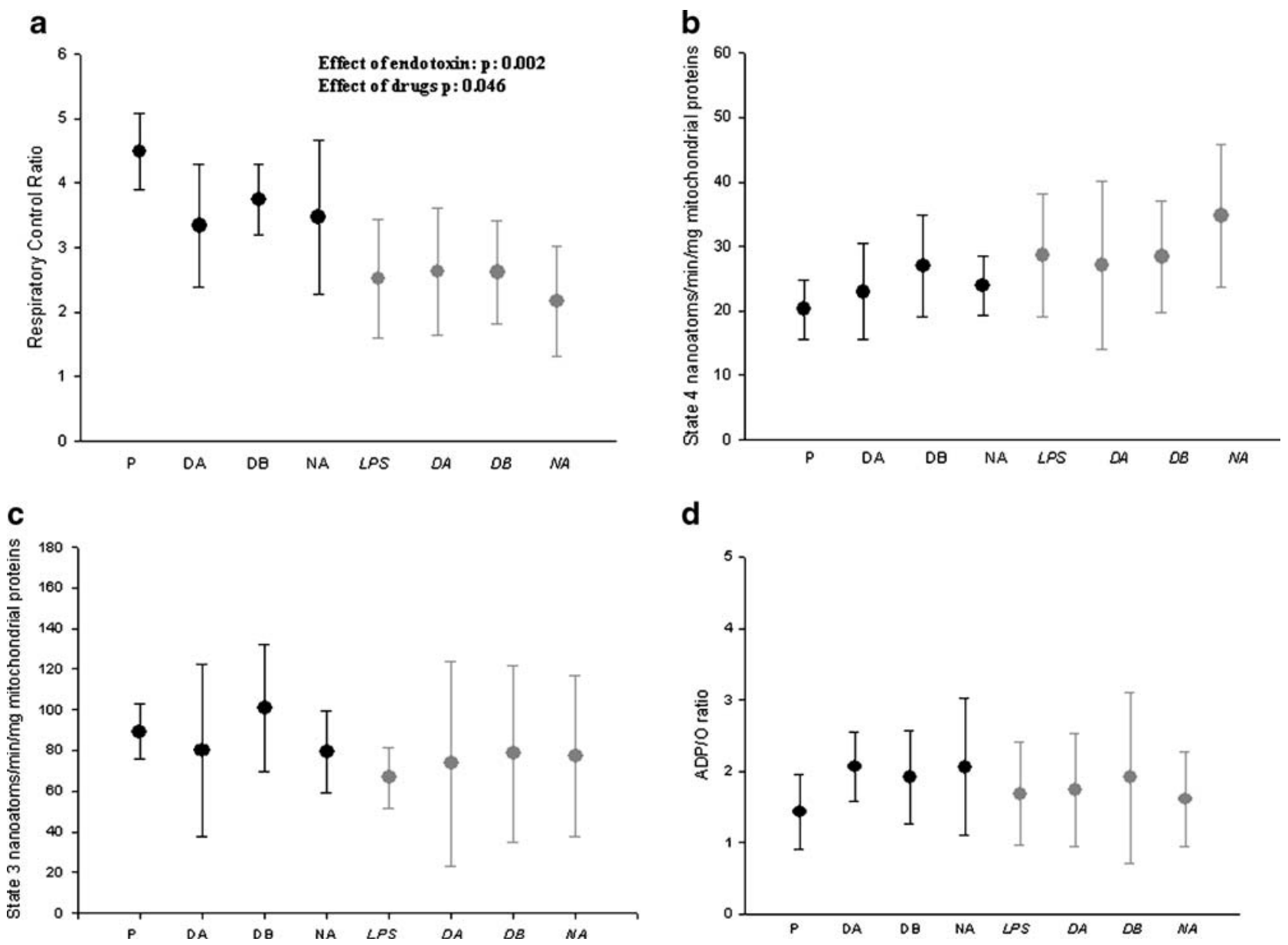

Fig. 2. a (upper left), b (upper right), $\mathbf{c}$ (lower left), and $\mathbf{d}$ (lower right). Changes in glutamate-dependent respiratory control ratio (RCR) (a), state 4 respiration (b), state 3 respiration (c), and ADP:O ratio (d), respectively, after endotoxin and catecholamine exposure. Data are indicated as mean and SD. $P$ placebo, LPS lipopolysaccharide, DA dopamine, DB dobutamine, NA noradrenaline. Dark symbols: placebo incubation; shaded symbols: LPS incubation. 
Table 1. State 4 Respiration Rates

\begin{tabular}{|c|c|c|c|c|c|c|c|c|}
\hline & $\mathrm{P} /-$ & $\mathrm{P} / \mathrm{DA}$ & $\mathrm{P} / \mathrm{DB}$ & $\mathrm{P} / \mathrm{NA}$ & LPS/- & LPS/DA & LPS/DB & LPS/NA \\
\hline Complex II (succinate) & $61 \pm 35$ & $46 \pm 23$ & $54 \pm 29$ & $43 \pm 29$ & $42 \pm 17$ & $44 \pm 25$ & $47 \pm 21$ & $53 \pm 25$ \\
\hline Complex IV (ascorbate/TMPD) & $64 \pm 24$ & $64 \pm 26$ & $73 \pm 11$ & $70 \pm 40$ & $50 \pm 32$ & $64 \pm 20$ & $77 \pm 23$ & $66 \pm 21$ \\
\hline
\end{tabular}

Values are nanomol $\mathrm{O}_{2} / \mathrm{min} / \mathrm{mg}$ mitochondrial protein. Data are expressed as mean $\pm \mathrm{SD} . \quad N=5$ (P/NA, Complex II; LPS/-; LPS/DB, Complex IV), otherwise, $n=6$

$P$ placebo, $L P S$ lipopolysaccharide, $D A$ dopamine, $D B$ dobutamine, $N A$ noradrenaline

respiratory activity was completed at most $5 \mathrm{~h}$ later (for all groups).

\section{Statistical Analysis}

The SPSS 11.0 software package was used for statistical analysis (SPSS Inc., Chicago, IL, USA). To evaluate the effects of endotoxin and catecholamines on mitochondrial function, analysis of variance for repeated measurements (MANOVA) was performed, using two within-subject factors (endotoxin/placebo [two levels] and drugs [four levels]). Statistical significance was considered at $P<0.05$. Data are expressed as mean $(\mathrm{SD})$.

\section{RESULTS}

Altogether, 48 samples were analyzed. One measurement of succinate-dependent respiration and one of ascorbate/TMPD-dependent respiration were not performed for technical reasons. One additional measurement of ascorbate/TMPD-dependent respiration could not be used because state 4 respiration was not clearly detectable; consequently, the RCR could not be calculated.

Dopamine, dobutamine and noradrenaline decreased glutamate-dependent RCR (drug effect, $p$ : 0.046; Fig. 2a). All catecholamines tended to increase the glutamate-dependent state 4 respiration rate in placebo samples $[18.4 \pm 3.1$ (control group) vs. $23.0 \pm$
7.4 (dopamine), $27.0 \pm 7.9$ (dobutamine), and $24.0 \pm 4.7$ (noradrenaline)], but the effects were not significant (Fig. 2b). None of the drugs changed succinate-dependent or ascorbate/TMPD-dependent state 4 respiration rates (Table 1), and none of the drugs affected state 3 respiration in any of the complexes of the mitochondrial respiratory chain (Fig. 2c, Table 2).

The glutamate-dependent respiratory control ratio (RCR; state 3/state 4) was reduced by endotoxin $[4.5 \pm$ 0.6 (control group) vs. $2.5 \pm 0.9$ (endotoxin group); $p=$ 0.002 , Fig. 2a], but the other RCRs were not affected (Table 3). Endotoxin tended to decrease glutamatedependent state 3 respiration [89.3 \pm 13.7 (control group) vs. $66.6 \pm 14.7$ (endotoxin group)], but the effect was not significant (Fig. 2c). Succinate and ascorbate/TMPDdependent state 3 respiration were not affected by endotoxin (Table 2). Endotoxin was associated with a slight but insignificant increase in glutamate-dependent state 4 respiration [20.2 \pm 4.6 (control group) vs. $28.5 \pm 9.5$ (endotoxin group); $p=0.1$, Fig. 2b]. Succinate and ascorbate/TMPD-dependent state 4 respiration rates were not affected by endotoxin (Table 1).

None of the drugs affected the substrate-dependent state 3 and 4 respiration rates in samples previously exposed to endotoxin, and all drugs failed to restore the endotoxin-induced decrease in glutamate-dependent RCR (Fig. 2a). Similarly, none of the drugs altered the other substrate-dependent RCRs (Table 3).

As shown in Table 4 and Fig. 2d, the ADP:O ratios and the maximal ATP production of the two analyzed

Table 2. State 3 Respiration Rates

\begin{tabular}{|c|c|c|c|c|c|c|c|c|}
\hline & $\mathrm{P} /-$ & $\mathrm{P} / \mathrm{DA}$ & $\mathrm{P} / \mathrm{DB}$ & $\mathrm{P} / \mathrm{NA}$ & LPS/- & LPS/DA & LPS/DB & LPS/NA \\
\hline Complex II (succinate) & $182 \pm 86$ & $190 \pm 104$ & $197 \pm 96$ & $178 \pm 111$ & $135 \pm 72$ & $155 \pm 115$ & $139 \pm 84$ & $172 \pm 120$ \\
\hline Complex IV (ascorbate/TMPD) & $181 \pm 68$ & $164 \pm 87$ & $185 \pm 52$ & $155 \pm 91$ & $123 \pm 83$ & $138 \pm 71$ & $233 \pm 158$ & $142 \pm 72$ \\
\hline
\end{tabular}

Values are nanomol $\mathrm{O}_{2} / \mathrm{min} / \mathrm{mg}$ mitochondrial protein. Data are expressed as mean $\pm \mathrm{SD}$. $N=5$ (P/NA, Complex II; LPS/-; LPS/DB, Complex IV), otherwise, $n=6$

$P$ placebo, $L P S$ lipopolysaccharide, $D A$ dopamine, $D B$ dobutamine, $N A$ noradrenaline 
Table 3. Respiratory Control Ratios

\begin{tabular}{|c|c|c|c|c|c|c|c|c|}
\hline & $\mathrm{P} /-$ & $\mathrm{P} / \mathrm{DA}$ & $\mathrm{P} / \mathrm{DB}$ & $\mathrm{P} / \mathrm{NA}$ & LPS/- & LPS/DA & LPS/DB & LPS/NA \\
\hline Complex II (succinate) & $3.3 \pm 1.4$ & $4.1 \pm 0.6$ & $3.9 \pm 0.8$ & $4.2 \pm 1.7$ & $3.2 \pm 1.2$ & $3.4 \pm 1.5$ & $3.0 \pm 1.1$ & $3.1 \pm 1.1$ \\
\hline Complex IV (ascorbate/TMPD) & $2.9 \pm 0.8$ & $2.5 \pm 0.3$ & $2.5 \pm 0.5$ & $2.2 \pm 0.6$ & $2.5 \pm 0.8$ & $2.0 \pm 0.5$ & $2.3 \pm 0.8$ & $2.1 \pm 0.5$ \\
\hline
\end{tabular}

Data are expressed as mean \pm SD. $N=5$ (P/NA, Complex II; LPS/-; LPS/DB, Complex IV), otherwise, $n=6$

$P$ placebo, $L P S$ lipopolysaccharide, $D A$ dopamine, $D B$ dobutamine, $N A$ noradrenaline

mitochondrial complexes were not affected by catecholamines or endotoxin.

\section{DISCUSSION}

In this study, all catecholamines impaired the efficiency of glutamate-dependent liver mitochondrial respiration. While endotoxin alone had a similar effect, catecholamine administration to endotoxin-exposed samples resulted in neither an additive nor a beneficial effect on mitochondrial respiration.

The effect of catecholamines, and in particular of dopamine, on mitochondrial function confirms results published by others [3, 4]. Ben-Shachar et al. demonstrated that dopamine inhibits mitochondrial enzyme complex I activity and reduces tissue ATP concentration in the brain [3], while Berman and Hastings found dopamine-induced increases in resting state 4 respiration in the brain and in the liver [4]. This seemed to be the consequence of quinine-induced membrane swelling [4]. Nevertheless, stimulation of adrenoreceptors on the cell membrane can also trigger metabolic effects which will have a major secondary influence on mitochondrial energy production. A number of papers have demonstrated that catecholamines can induce changes in mitochondrial activity, both at the level of Krebs' cycle and in the electron transport chain [20, 23]. Dopamine, dobutamine and noradrenalin have a similar chemical structure (dihydroxyphenyl-group), and all of them stimulate thus alpha- and beta-receptors, although to various degrees. It has been shown that isoproterenol interferes with mitochondrial enzyme-activity in isolated mitochondria, and decreases respiratory control ratio [20]. We hypothesize that the consistent role in hepatic mitochondrial damage is related to the similar chemical structure of the three drugs.

We reported previously that catecholamines are able to partially reverse endotoxin-induced alterations in mitochondrial function [21]. Since the technique we used to perform the experiments was the same, the discrepancy between our previous study and the present study seems to be related to different organ sensitivity to catecholamine and endotoxin exposure. Prolonged anesthesia (12 h, vs. $30 \mathrm{~min}$ in the previous series) may offer an alternative explanation. We found recently that state 4 but not state 3 glutamate-dependent respiration was altered $24 \mathrm{~h}$ after in vivo exposure to endotoxin [22]. In rat left ventricular myocardium, volatile anesthetics evoked prolonged changes in the proteome, including proteins of mitochondrial respiration [14]. However, we used intravenous anesthetics in contrast to volatile anesthetics.

Sepsis-induced complex I dysfunction has been demonstrated during both experimental and clinical sepsis $[5,6,9,19,22]$. In a recent study, endotoxin induced significant alterations in state 4 but not state 3 respiration in muscle mitochondria [21]. These alterations were partially reversed by dopamine and dobutamine, and the ADOP:O ratio increased, suggesting that the drugs increased the amount of energy that could be produced per unit of oxygen consumed in endotoxinexposed mitochondria.

Table 4. ADP:O Ratio and Maximal ATP Production

\begin{tabular}{|c|c|c|c|c|c|c|c|c|c|}
\hline & & $\mathrm{P} /-$ & $\mathrm{P} / \mathrm{DA}$ & $\mathrm{P} / \mathrm{DB}$ & $\mathrm{P} / \mathrm{NA}$ & LPS/- & LPS/DA & LPS/DB & LPS/NA \\
\hline Complex I (glutamate) & Maximal ATP production & $130 \pm 60$ & $155 \pm 78$ & $199 \pm 106$ & $148 \pm 25$ & $107 \pm 38$ & $143 \pm 143$ & $146 \pm 117$ & $121 \pm 68$ \\
\hline Complex II (succinate) & $\mathrm{ADP} / \mathrm{O}$ ratio & $1.2 \pm 0.2$ & $1.5 \pm 0.4$ & $1.3 \pm 0.2$ & $2 \pm 0.5$ & $1.2 \pm 0.4$ & $1.7 \pm 0.9$ & $1.2 \pm 0.4$ & $1.2 \pm 0.6$ \\
\hline & maximal ATP production & $235 \pm 125$ & $279 \pm 166$ & $254 \pm 120$ & $249 \pm 156$ & $172 \pm 120$ & $217 \pm 185$ & $171 \pm 139$ & $220 \pm 230$ \\
\hline
\end{tabular}

Values are nanomol/nanoatom (ADP:O ratios) and nanomol $\times$ nanoatom $/ \mathrm{min} / \mathrm{mg}$ protein (ATP production). Data are expressed as mean $\pm \mathrm{SD} . N=5(\mathrm{P} /$ NA, ADP/O ratio; P/NA, maximal ATP production), otherwise, $n=6$

$P$ placebo, $L P S$ lipopolysaccharide, $D A$ dopamine, $D B$ dobutamine, $N A$ noradrenaline, $A D P$ adenosine diphosphate, $A T P$ adenosine triphosphate 
Several investigators have demonstrated organ-specific differences in mitochondrial response during experimental sepsis. For example, cardiac muscle mitochondria seemed to be more sensitive to endotoxin than skeletal muscle [26]. And whereas endotoxin induced ATP depletion in liver and kidney samples, this was not the case in skeletal muscle samples [27]. Furthermore, significant differences with respect to respiratory activity in state 3 and ADP-to-oxygen ratio were found between heart muscle and liver mitochondria in subgroups of rats exposed to endotoxin [16].

This in vitro experiment has limitations, since it does not reflect the corresponding in vivo conditions. The removal of the mitochondria from their physiological surroundings and the short time period of incubation essentially avoid the effects of inflammatory mediators, hormonal changes, and enzyme induction, which can indirectly influence mitochondrial function. However, our model eliminates circulatory abnormalities and systemic effects of endotoxin on oxygen metabolism and demonstrates the direct effects on mitochondria, which have also been described by others $[2,6,18]$. Additional studies investigating the effects of such substances on isolated cells, on tissue samples, and directly in vivo may provide further information on the effects of commonly used drugs and inflammatory states on cellular functions.

In conclusion, we demonstrate that catecholamines impair the efficiency of glutamate-dependent liver mitochondrial respiration in vitro. These effects were not demonstrated in endotoxin-incubated mitochondria. Our findings, together with results from other studies, suggest that catecholamines may have organ- and disease-specific effects on mitochondrial respiration.

\section{ACKNOWLEDGEMENT}

Supported by grant $3200 \mathrm{BO} / 102268$ from the Swiss National Fund.

\section{REFERENCES}

1. Ainscow, E. K., and M. D. Brand. 1999. The responses of rat hepatocytes to glucagon and adrenaline. Application of quantified elasticity analysis. Eur. J. Biochem. 265:1043-1055.

2. Antunes, F., D. Han, D. Rettori, and E. Cadenas. 2002. Mitochondrial damage by nitric oxide is potentiated by dopamine in PC12 cells. Biochim. Biophys. Acta. 1556:233-238.

3. Ben-Shachar, D., R. Zuk, H. Gazawi, and P. Ljubuncic. 2004. Dopamine toxicity involves mitochondrial complex I inhibition: implications to dopamine-related neuropsychiatric disorders. Biochem. Pharmacol. 67:1965-1974.
4. Berman, S. B., and T. G. Hastings. 1999. Dopamine oxidation alters mitochondrial respiration and induces permeability transition in brain mitochondria: implications for Parkinson's disease. $J$. Neurochem. 73:1127-1137.

5. Brealey, D., S. Karyampudi, T. S. Jacques, M. Novelli, R. Stidwill, V. Taylor, R. T. Smolenski, and M. Singer. 2004. Mitochondrial dysfunction in a long-term rodent model of sepsis and organ failure. Am. J. Physiol. Regul. Integr. Comp. Physiol. 286:R491R497.

6. Brealey, D., and M. Singer. 2003. Mitochondrial dysfunction in sepsis. Curr. Infect. Dis. Rep. 5:365-371.

7. Carvalho, M., F. Remiao, N. Milhazes, F. Borges, E. Fernandes, F. Carvalho, and M. L. Bastos. 2004. The toxicity of N-methyl-alphamethyldopamine to freshly isolated rat hepatocytes is prevented by ascorbic acid and N-acetylcysteine. Toxicology. 200:193-203.

8. Chance, B., and G. R. Williams. 1956. Respiratory enzymes in oxidative phosphorylation. VI. The effects of adenosine diphosphate on azide-treated mitochondria. J. Biol. Chem. 221:477-489.

9. Crouser, E. D., M. W. Julian, D. V. Blaho, and D. R. Pfeiffer. 2002. Endotoxin-induced mitochondrial damage correlates with impaired respiratory activity. Crit. Care Med. 30:276-284.

10. Estabrook, R. W. 1967. Mitochondrial respiratory control and the polarographic measurement of ADP:O ratios. In: Methods in Enzymology, Vol. X, Oxidation and Phosphorylation, Kaplan, NP, NP Colowick, RW Estabrook, and ME Oullman, eds. Academic, New York, pp. 41-47.

11. Gorrasi, J., V. Krejci, L. Hiltebrand, S. Brand, H. Bracht, B. Balsiger, J. Takala, and S. Jakob. 2007. Apparent heterogeneity in splanchnic vascular response to norepinephrine during sepsis. Crit. Care. 11:S14.

12. Jakob, S. M., E. Ruokonen, and J. Takala. 2002. Effects of dopamine on systemic and regional blood flow and metabolism in septic and cardiac surgery patients. Shock. 18:8-13.

13. Johnson, D., and H. A. Lardy. 1967. Isolation of liver or kidney mitochondria. In: Methods in Enzymology, Vol. X, Oxidation and Phosphorylation, Kaplan, NP, NP Colowick, , RW Estabrook, , and ME Oullman, eds. Academic, New York, pp. 94-96.

14. Kalenka, A., M. H. Maurer, R. E. Feldmann, W. Kuschuinsky, and K. F. Waschke. 2006. Volatile anesthetics evoke prolonged changes in the proteome of the left ventricule myocardium: defining a molecular basis of cardioprotection. Acta Anaesthesiol. Scand. 50:414- 427.

15. Knuesel, R., H. Bracht, V. Krejci, S. Z. Ali, L. Bruegger, J. Takala, and S. M. Jakob. 2005. High peritoneal lactate concentration after surgery and in sepsis detected by membrane microdialysis. Intensive Care Med. 31:S208.

16. Kozlov, A. V., K. Staniek, S. Haindl, C. Piskernik, W. Ohlinger, L. Gille, H. Nohl, S. Bahrami, and H. Redl. 2006. Different effects of endotoxic shock on the respiratory function of liver and heart mitochondria in rats. Am. J. Physiol. Gastrointest. Liver Physiol. 290:G543-G549.

17. Krahenbuhl, S., J. Stucki, and J. Reichen. 1989. Mitochondrial function in carbon tetrachloride-induced cirrhosis in the rat. Qualitative and quantitative defects. Biochem. Pharmacol. 38:1583-1588.

18. McGivney, A., and S. G. Bradley. 1980. Action of bacterial lipopolysaccharide on the respiration of mouse liver mitochondria. Infect. Immun. 27:102-106.

19. Mela, L., L. V. Bacalzo Jr., and L. D. Miller. 1971. Defective oxidative metabolism of rat liver mitochondria in hemorrhagic and endotoxin shock. Am. J. Physiol. 220:571-577.

20. Poderoso, J. J., S. Fernandez, M. C. Carreras, C. G. Del Bosco, and A. Boveris. 1995. Isoproterenol-dependent decrease in oxygen uptake and respiratory enzyme activities in rat myocardial tissue and mitochondria. Crit. Care Med. 23:1726-1733.

21. Porta, F., J. Takala, C. Weikert, P. Kaufmann, S. Krahenbuhl, and S. M. Jakob. 2006a. Effect of endotoxin, dobutamine and dopamine on muscle mitochondrial respiration in vitro. $J$. Endotoxin Res. 12:358-366. 
22. Porta, F., J. Takala, C. Weikert, H. Bracht, A. Kolarova, B. H. Lauterburg, E. Borotto, and S. M. Jakob. 2006b. Effects of prolonged endotoxemia on liver, skeletal muscle and kidney mitochondrial function. Crit. Care. 10:R118.

23. Shukla, V. H., K. R. Dave, and S. S. Katyare. 2000. Effect of catecholamine depletion on oxidative energy metabolism in rat liver, brain and heart mitochondria; use of reserpine. Сomp. Biochem. Physiol., Toxicol. Pharmacol. 127:79-90.

24. Sivaramakrishnan, S., and T. Ramasarma. 1983. Noradrenaline stimulates succinate dehydrogenase through beta-adrenergic receptors. Indian J. Biochem. Biophys. 20:16-22.
25. Tovar, L., C. Weikert, B. Balsiger, L. Bruegger, G. Beldi, J. Takala, and S. M. Jakob. 2005. Decreased in-vitro sensitivity to norepinephrine after endotoxin-exposure and faecal peritonitis. Intensive Care Med. 31:S17.

26. Trumbeckaite, S., J. R. Opalka, C. Neuhof, S. Zierz, and F. N. Gellerich. 2001. Different sensitivity of rabbit heart and skeletal muscle to endotoxin-induced impairment of mitochondrial function. Eur. J. Biochem. 268:1422-1429.

27. Van Lambalgen, A. A., A. A. van Kraats, M. F. Mulder, T. Teerlink, and G. C. van den Bos. 1994. High-energy phosphates in heart, liver, kidney, and skeletal muscle of endotoxemic rats. $\mathrm{Am}$. $J$. Physiol. 266:H1581-H1587. 